\title{
Unexpected complications related to tamponade after vitrectomy
}

\author{
Masahito Ohji ${ }^{1}$
}

Received: 24 May 2016 / Accepted: 1 June 2016 / Published online: 10 June 2016

(C) Springer-Verlag Berlin Heidelberg 2016

Vitrectomy is one of the most important and most commonly performed surgeries among ocular surgeries. Many adjunctives are used during vitrectomy including air, gases, silicone oil, perfluorocarbon liquid, triamcinolone acetonide, and dyes. All adjunctives are useful and indispensable tools to achieve safer procedures and better outcomes; however, each adjunctive may cause complications. Some adjunctives may cause complications commonly and some rarely, some complications may be serious and some may be easy to manage. Vitreo-retinal surgeons should understand their safety and complications and be cautious when they use them. Especially, surgeon should pay attention to tamponade materials because they stay in the vitreous cavity for week or months or even permanently.

Silicone oil and gases are the major indispensable materials for tamponade during vitrectomy. Silicone oil can be used in regular retinal detachment but are especially important in complicated retinal detachment or proliferative vitreoretinopathy because of long-lasting tamponade effect. Well-known complications of silicone oil include cataract, glaucoma, keratopathy, and epiretinal membrane formation, while migration into brain and visual loss are relatively rare among the complications caused by silicone oil tamponade [1-3]. Many surgeons may be aware of the occurrence of visual loss after silicon oil tamponade without finding specific reasons, while several case series with visual loss after silicone oil tamponade have been published. However, it might be difficult to conclude that the

Masahito Ohji

ohji@belle.shiga-med.ac.jp

1 Department of Ophthalmology, Shiga University of Medical Science, Otsu, Japan visual loss is caused by silicone oil tamponade, because silicone oil is mainly used in complicated cases. The cause of visual loss could be attributed to pre-operative conditions such as maculaoff retinal detachment. Therefore, it is difficult to know the incidence of visual loss caused by silicone oil tamponade.

Tode et al. retrospectively analyzed 900 cases with silicone oil tamponade out of 5,400 cases with vitrectomy [1]. They selected 15 cases who had a rhegmatogenous retinal detachment with an attached macula before, during and after vitrectomy out of 900 cases. Vision loss with central scotoma developed in eight patients out of 15 cases and visual acuity did not recover, and remained 20/200 in three cases even years after oil removal. In addition to the functional deterioration, OCT revealed significant reduction of the thickness of the nerve fiber, ganglion cells, and inner plexiform layers compared with their fellow eyes. These changes in the retina were not found in cases without visual loss. Christensen et al. reported that profound visual loss worse than 6/60 was identified in three of nine eyes with macula-on rhegmatogenous retinal detachment after silicone oil tamponade, but such profound visual loss did not develop in any of the patients with gas tamponade [2]. The incidence of profound visual loss in eyes with macula-on retinal detachment treated with silicone oil tamponade ranged from $20 \%$ to $33 \%$. The incidence of serious visual loss after uncomplicated vitrectomy and silicone oil tamponade may be higher than we have believed. The retinal thinning in OCT, especially in the inner retinal layer, and abnormal ERG findings has been reported, but the mechanism of visual loss caused by silicone oil tamponade is not well elucidated, and risk factors and prevention are not established. As described, silicone oil tamponade may cause serious retinal damage and profound visual loss in eyes with macula-on retinal detachment. It would be reasonable to think that similar damage would develop in eyes with macular-off retinal detachment or other pathology after silicone oil tamponade, although it might be difficult to evaluate the damage 
because of underlying pathology. Obviously, surgeons also need to pay attention to other complications related to silicone oil tamponade. Increase of intraocular pressure is a wellknown most frequent complication. Migration of silicone oil into the cerebral ventricular system probably through the optic nerve is a rare complication, while systemic symptoms seem to be rare [4].

Gases are other important materials for tamponade. Serious visual loss caused by gas tamponade may be less than for silicone oil tamponade; however, gas also has complications specific to it. Increase of intraocular pressure is one of the most important and common complications associated with gas tamponade. Because injection of pure $\mathrm{SF}_{6}$ or pure $\mathrm{C}_{3} \mathrm{~F}_{8}$ causes increase of IOP, so appropriately diluted gases should be used as tamponade during vitrectomy $[5,6]$. Physicians need to pay attention to gases until they have disappeared form the vitreous cavity completely, because even a small amount of intraocular gas may increase intraocular pressure significantly in certain situations. IOP may significantly increase and result in transient or permanent blindness when general anesthesia using nitrous oxide gas is performed [7]. Patients may not able to explain that they have intraocular gas because of consciousness disturbance by accidents. Intraocular pressure may also increase when patients move into an atmosphere with low air pressure, including air or even train travel $[8,9]$. To prevent these serious complications, the risk of intraocular pressure increase has to be understood by patients, ophthalmologists, and anesthesiologists. Therefore, a wrist band showing the use of intraocular gas should be used until complete disappearance of intraocular gas, to let physicians know it is being used.

Fluid-air exchange is performed before gas tamponade or silicone oil tamponade in most cases. Even a procedure of fluid-air exchange may cause visual field constriction, possibly due to dehydration of the retina or direct mechanical damage of the retina caused by direct air flow [10]. In particular, the trocar system without valve in small-gauge vitrectomy may permit a large flow of air and cause retinal damage at the opposite side of the infusion, and may also cause a constriction of the visual field [11]. This complication could be prevented by humidifying air, lowering air pressure, or using valved cannula $[12,13]$.
Materials for tamponade including silicone oil and gases are indispensable for modern vitreoretinal surgery, and some new materials may be introduced to vitreoretinal surgeries in future. Surgeons should bear in mind that any materials or procedures may cause serious complications.

\section{References}

1. Tode J, Purtskhvanidze K, Oppermann T, Hillenkamp J, Treumer F, Roider J. (2016) Vision loss under silicone oil tamponade. Retina [in press]

2. Christensen UC, Cour ML (2012) Visual loss after use of intraocular silicone oil associated with thinning of inner retinal layers. Acta Ophthalmol 90:733-737

3. Cox MS, Azen SP, Barr CC, Linton KL, Diddie KR, Lai MY, Freeman HM, Irvine A (1995) Macular pucker after successful surgery for proliferative vitreoretinopathy. Silicone Study Report 8. Ophthalmology 102:1884-1891

4. Eller AW, Friberg TR, Mah F (2000) Migration of silicone oil into the brain: a complication of intraocular silicone oil for retinal tamponade. Am J Ophthalmol 129:685-688

5. Abrams GW, Edelhauser HF, Aaberg TM, Hamilton LH (1974) Dynamics of intravitreal sulfur hexafluoride gas. Invest Ophthalmol 13:863-868

6. Peters MA, Abrams GW, Hamilton LH, Burke JM, Schrieber TM (1985) The nonexpansile equilibrated concentration of perfluoropropane gas in the eye. Am J Ophthalmol 100:831-839

7. Wolf GL, Capuano C, Hartung J (1983) Nitrous oxide increases intraocular pressure after intravitreal sulfur hexafluoride injection. Anesthesiology 59:547-548

8. Lincoff H, Weinberger D, Stergiu P (1989) Air travel with intraocular gases. II. Clinical considerations. Arch Ophthalmol 107:907910

9. Shiramizu KM, Okada AA, Hirakata A (2001) Transient amaurosis associated with intraocular gas during ascending high-speed train travel. Retina 21:528-529

10. Melberg NS, Thomas MA (1995) Visual field loss after pars plana vitrectomy with air/fluid exchange. Am J Ophthalmol 120:386-388

11. Machado LM, Magalhaes O Jr, Maia M, Rodorigues EB, Farah ME, Ismail KA, Molon L, Oliveira DA (2011) Comparison of 20-, 23-, and 25-gauge air infusion forces. Retina 31:2002-2006

12. Ohji M, Nao-I N, Saito Y, Hayashi A, Tano Y (1999) Prevention of visual field defect after macular hole surgery by passing air used for fluid-air exchange through water. Am J Ophthalmol 127:62-66

13. Hirata A, Yonemura N, Hasumura T, Murata Y, Negi A (2000) Effect of infusion air pressure on visual field defects after macular hole surgery. Am J Ophthalmol 130:611-616 